\title{
JOGADORES JUVENIS DE FUTEBOL SUBMETIDOS A TREINAMENTO GENERALISTAE DIFERENÇA NA POTÊNCIA AERÓBIA EM CONSEQUÊNCIA DE SEU POSICIONAMENTO TÁTICO EM CAMPO
}

\author{
Marcelo Ribeiro Ramos, Faculdade de Minas - FAMINAS, Muriaé, Minas Gerais - Brasil \\ Cristiano Andrade Quintão Coelho Rocha, Faculdade de Minas - FAMINAS, Muriaé, Minas \\ Gerais - Brasil \\ Jairo Antônio da Paixão, Universidade Federal de Ouro Preto - UFOP, Minas Gerais - Brasil \\ Guilherme Tucher, Faculdade de Minas - FAMINAS, Muriaé, Minas Gerais - Brasil
}

\section{RESUMO}

$\mathrm{O}$ presente estudo objetivou analisar se há diferença no $\mathrm{VO}_{2}$ máximo de jogadores juvenis de futebol em decorrência de seu posicionamento tático em jogo apesar de realizarem um treinamento físico generalista. Participaram desse estudo 27 futebolistas do sexo masculino da categoria juvenil de um clube de futebol com experiência competitiva de mais de dois anos na modalidade. O grupo foi dividido em zagueiros $(\mathrm{G} 1 ; \mathrm{n}=8)$, meio campistas e laterais $(\mathrm{G} 2$; $\mathrm{n}=10)$ e, finalmente, atacantes $(\mathrm{G} 3 ; \mathrm{n}=9)$. Para o desenvolvimento físico dos atletas, não houve especificação do treinamento entre as funções táticas assumidas em jogo. Torneios de menor importância também foram utilizados como forma de treinamento no início da temporada. $\mathrm{O}$ $\mathrm{VO}_{2}$ máx foi avaliado através do teste de corrida de vai-e-vem aplicado no próprio campo de treinamento. Encontrou-se diferença significativa entre o $\mathrm{VO}_{2}$ máx de G1 e G2 (p=0,002) e entre G2 e G3 ( $p=0,003)$. Não foi encontrada diferença no $\mathrm{VO}_{2}$ máx entre $\mathrm{G} 1$ e $\mathrm{G} 3$ ( $\mathrm{p}=$ $0,938)$. Os meio campistas e laterais apresentaram maior $\mathrm{VO}_{2}$ máximo mesmo que a condução do treinamento não privilegie diferenças em decorrência de sua função tática em campo.

Palavras-Chave: Futebol; Consumo de oxigênio; Tática; Desempenho físico.

\section{GENERAL TRAINING OF YOUTH SOCCER PLAYER AND DIFFERENCES IN AEROBIC POWER IN CONSEQUENCE OF HIS TACTICAL POSITION}

\begin{abstract}
The study aimed to evaluate the differences in $\mathrm{O}_{2}$ uptake of youth soccer players due to his tactical positioning game although his general training. The sample consisted of 27 male soccer players from the youth category of a same club. The group was divided into defenders $(\mathrm{G} 1, \mathrm{n}=8)$, midfielders and sides $(\mathrm{G} 2, \mathrm{n}=10)$, and forwards $(\mathrm{G} 3, \mathrm{n}=9)$. The $\mathrm{O}_{2}$ uptake was measured by multistage $20 \mathrm{~m}$ shuttle runapplied in own training camp. The results showed a significant difference between $\mathrm{O}_{2}$ uptake of G1 and G2 $(\mathrm{p}=0.002)$ and between G2 and G3 (p $=0.003)$. No difference was found in $\mathrm{O}_{2}$ uptake between $\mathrm{G} 1$ and $\mathrm{G} 3(\mathrm{p}=0.938)$. The midfielders and side players had higher $\mathrm{O}_{2}$ uptake even if the management of the training focused not due to differences in their tactics depending on the field.
\end{abstract}


Key-Words: Soccer; Oxygen uptake, Tactics, Physical performance. 


\section{INTRODUÇÃO}

O futebol é uma modalidade esportiva com exercícios intermitentes de intensidade variável. ${ }^{1,2}$ Diferentes estudos têm mostrado que o deslocamento dos jogadores durante as partidas é determinado principalmente pela posição ou função tática exercida. ${ }^{3}$ Durante um jogo de futebol, os jogadores percorrem aproximadamente de 10 a $13 \mathrm{~km}$, sendo que os meio campistas são responsáveis pelas maiores distâncias. ${ }^{4}$ Alguns autores apontam que a distância e a intensidade de corrida no primeiro tempo é maior que a do segundo tempo. ${ }^{3,5}$ Outros só encontraram esta diferença para a distância corrida em intensidade média. ${ }^{6}$ Assim, o condicionamento físico no futebol, representa um pré-requisito para a performance técnica, tática e psíquica na competição. ${ }^{7} \mathrm{O}$ futebolista, dada a natureza intermitente do seu esforço e a ampla faixa de intensidades que o caracteriza, tem de privilegiar no seu treino aspectos distintos como o desenvolvimento da potência, da velocidade, da resistência anaeróbia e da resistência aeróbia. ${ }^{8}$ Entre as várias capacidades físicas utilizadas por um atleta de futebol, pode-se destacar a aeróbia. Sua manifestação tem importância durante a partida e no período destinado à recuperação dos jogadores, garantindo maiores estoques de glicogênio muscular. ${ }^{9}$

A potência aeróbia máxima $\left(\mathrm{VO}_{2}\right.$ máx) tem sido de grande valia na avaliação de jogadores de futebol, ${ }^{10}$ pois é a variável fisiológica que melhor descreve a capacidade funcional dos sistemas cardiovascular e respiratório. ${ }^{11}$ É aceito como o índice que representa a capacidade máxima de integração do organismo em captar, transportar e utilizar o oxigênio para os processos aeróbios de produção de energia durante a contração muscular. ${ }^{12} \mathrm{O} \mathrm{VO}_{2}$ máxé considerado um dos parâmetros de grande importância na prediçãodo desempenho, pois a capacidade do ser humano em realizar exercícios de média e longa duração depende principalmente do metabolismo aeróbio sendo, assim, um índice muito empregado para classificar a capacidade funcional cardiorrespiratória, sobretudo em atletas. ${ }^{9,13} \mathrm{O} \mathrm{VO}_{2}$ máx tem tendência de apresentar valores mais elevados quanto maior for a importância do metabolismo aeróbio no desempenho de cada modalidade. ${ }^{13}$

Nas modalidades esportivas coletivas, os valores de $\mathrm{VO}_{2}$ máx encontram-se mais baixos quando comparados a modalidades esportivas individuais. Foram encontrados valores de $51,15 \mathrm{ml} / \mathrm{min} / \mathrm{kg}$ no basquetebol, $51,41 \mathrm{ml} / \mathrm{min} / \mathrm{kg}$ no handebol, $55,12 \mathrm{ml} / \mathrm{min} / \mathrm{kg}$ no vôlei e $56,66 \mathrm{ml} / \mathrm{min} / \mathrm{kg}$ no futsal. Nos esportes individuais, encontram-se $59,15 \mathrm{ml} / \mathrm{min} / \mathrm{kg}$ nos 
corredores, $59,62 \mathrm{ml} / \mathrm{min} / \mathrm{kg}$ nos judocas, $63,25 \mathrm{ml} / \mathrm{min} / \mathrm{kg}$ nos triatletas e $69,65 \mathrm{ml} / \mathrm{min} / \mathrm{kg}$ nos ciclistas. ${ }^{13}$ No futebol, foramapontadas diferenças não significativas no $\mathrm{VO}_{2}$ máx entre as categorias profissionais $(50,21 \pm 3,21 \mathrm{ml} / \mathrm{min} / \mathrm{kg})$, juniores $(49,58 \pm 2,89 \mathrm{ml} / \mathrm{min} / \mathrm{kg})$ e juvenis $\left(49,53 \pm 2,90(\mathrm{ml} / \mathrm{min} / \mathrm{kg}){ }^{11}\right.$

O princípio da especificidade preconiza que sejam treinados os aspectos que apresentem relação direta com a competição, estrutura do movimento, tipos de esforços e natureza das tarefas. A preocupação está em viabilizar a maior transferência possível das aquisições conseguidas no treino para o contexto específico no jogo. ${ }^{14}$ Estudos apontam que os laterais e meio-campistas são os jogadores com maior desenvolvimento da potência aeróbia, seguidos pelos atacantes e zagueiros. ${ }^{8,15}$ Os laterais e meio campistas possuem essa melhor condição aeróbia em resposta as exigências de suas funções. Há uma relação significativa entre o consumo máximo de oxigênio e a distância percorrida em campo pelos jogadores. ${ }^{16} \mathrm{O}$ aumento e os distintos níveis de capacidade aeróbia em atletas de diferentes posições no futebol estão fortemente relacionados à evolução tática da modalidade em que os sistemas de jogo tornam os jogadores especialistas em algumas funções especificas. ${ }^{14}$

Percebe-se que o processo de treinamento de jogadores de futebol de alto rendimento é elaborado visando provocar adaptações específicas que atendam suas funções táticas e técnicas. ${ }^{14}$ Assim, nota-se uma diferença intencional entre a capacidade de força, velocidade e potência aeróbia dos jogadores em virtude de seu posicionamento. Entretanto, em atletas em formação, encontramos um treinamento com características mais generalistas, o que não justificaria possíveis diferenças na potência aeróbia, e de outras capacidades físicas, e conseqüentemente possíveis especificações táticas em campo.

Nessa perspectiva, este estudo objetivou comparar se há diferença no $\mathrm{VO}_{2}$ máximo de jogadores juvenis de futebol em decorrência de seu posicionamento em jogo apesar de realizarem um treinamento generalista que não privilegiaria diferenças no consumo de oxigênio. 


\section{MÉTODOS}

Foram avaliados 27 indivíduos pertencentes à categoria juvenil de um clube de futebol. Para realização dos experimentos, o grupo foi dividido intencionalmente de acordo com seu posicionamento tático em campo em 3 grupos. Em G1 $(n=8)$ foram incluídos zagueiros, em G2 ( $n=10)$ os meio campistas e laterais e em G3 (n=9) os atacantes. Essa divisão foi baseada na intensidade e distância de corrida percorrida pelos jogadores em campo e na necessidade de consumo de oxigênio em função das diferentes ações dos jogadores. Os zagueiros (G1) apresentaram idade média de 15,75 \pm 1,03 anos, massa corporal (MC) de 65,50 \pm 5,48 kg, estatura de 177,50 \pm 7,38 cm, índice de massa corporal (IMC) de $21,11 \pm 1,59 \mathrm{Kg} / \mathrm{m}^{2} \mathrm{e}$ percentual de gordura $(\% \mathrm{G})$ de $11,86 \pm 1,25 \%$. Em G2, a idade média foi de 16,30 $\pm 0,82$ anos, MC de 65,55 $\pm 5,38 \mathrm{~kg}$, estatura de 172,10 $\pm 6,47 \mathrm{~cm}$, IMC de 22,24 $\pm 2,11 \mathrm{Kg} / \mathrm{m}^{2}$ e $\% \mathrm{G}$ de 11,53 \pm 1,02\%. G3 apresentou idade média de 15,44 $\pm 1,13$ anos, MC de 60,50 \pm 7,62 kg, estatura de $170,44 \pm 6,18 \mathrm{~cm}$, IMC de $20,72 \pm 1,56 \mathrm{Kg} / \mathrm{m}^{2}$ e $\% \mathrm{G}$ de $12,30 \pm 1,78 \%$.

No início da temporada, a equipe realizou os treinamentos visando o campeonato estadual do Rio de Janeiro (categoria juvenil), competição alvo que ocorrera no segundo semestre de 2010. Os atletas treinavam cinco vezes por semana e em alguns momentos perfaziam até seis sessões semanais. A duração total do treinamento era de cerca de 90 minutos contando o aquecimento, a parte principal e o resfriamento. Todos os atletas possuíam experiência de mais de dois anos no futebol.

O treinamento dos jogadores foi dividido em treino físico, técnico e tático. O treinamento físico era composto de treino aeróbio, anaeróbio lático e alático. A ênfase no aeróbio foi dada no início da temporada e, aos poucos, diminuída, passando a ser treinado de forma secundária, com o objetivo somente de manutenção. Os atletas realizavam o mesmo treinamento físicodurante as sessões, não havendo distinção ou especificação entre as posições. Como forma de preparação, os atletas ainda competiram em torneios de menor importância no início da temporada.

Todos os responsáveis pelos atletas participantes assinaram o Termo de Consentimento Livre Esclarecido, aprovado pelo Comitê de Ética em Pesquisas da Faculdade de Minas sob o número 004/2010. 


\subsection{Avaliação antropométrica}

A bateria de testes iniciou com a mensuração da massa corporal $(\mathrm{kg})$ e da estatura $(\mathrm{cm}), \mathrm{com}$ os atletas vestindo a própria roupa de treino e descalços, por meio de uma balança eletrônica (Toledo® 2096 PP/2) com estadiômetro fixado à mesma. Para obtenção dos valores do índice de massa corporal (IMC), utilizou-se o cálculo da razão da massa corporal (Kg) pela medida da estatura $(\mathrm{m})$ elevada ao quadrado $\left(\mathrm{IMC}=\right.$ peso $\left./ \mathrm{m}^{2}\right)$. O protocolo de Faulkner foi utilizado para a obtenção do percentual de gordura corporal (dobras tricipital, subescapular, supra-ilíaca e abdominal) utilizando um adipômetro científico Sanny®.

\subsection{Avaliação da capacidade aeróbica}

O teste de corrida de vai-e-vem ${ }^{17}$ foi aplicado no próprio campo de treinamento, com os atletas utilizando sua vestimenta habitual.O teste foi antecedido por um aquecimento de 10 minutos, quando foram realizados exercícios dinâmicos e alongamentos de membros inferiores e superiores.Avaliaram-se entre 6 (seis) a 10 (dez) jogadores por vez. Os atletas correram em um espaço de 20 metros delimitado por duas linhas paralelas em um ritmo cadenciado enviado por sinal sonoro (bips) gravado em um CD especialmente para este fim. Os bips sonoros aconteceram a intervalos específicos para cada estágio, sendo que a cada bip o avaliado deveria cruzar com um dos pés uma das linhas indicativas dos 20 metros de corrida do teste. Ou seja, saindo de uma das linhas corre em direção a outra, cruza esta com pelo menos um dos pés ao ouvir um bip e volta em sentido contrário. No CD, o término de um estágio é sinalizado com dois bips consecutivos e com uma voz avisando o número do estágio concluído. A duração do teste depende da aptidão cardiorrespiratória do avaliado, sendo máximo e progressivo, menos intenso no início e mais intenso no final, perfazendo um total possível de 21 (vinte e um) minutos (estágios). Com o resultado doúltimo estágio completo determina-se de forma indireta o consumo máximo de oxigênio $\left(\mathrm{VO}_{2} \mathrm{máx}\right)$, por meio da equação: $\mathrm{VO}_{2}$ máx $=31,025+(3,238 \times \mathrm{V})-(3,248 \times \mathrm{I})+[(0.1536 \times \mathrm{V} \times \mathrm{I})] ;$ onde $\mathrm{v}=$ velocidade $(\mathrm{km} / \mathrm{h}) \mathrm{e} \mathrm{I}=$ idade em anos. $^{17}$

\subsection{Análise estatística}

Os dados foram descritos por meio da média e do desvio padrão (média \pm desvio padrão). Utilizou-se do teste de Shapiro Wilk para verificar a normalidade da amostra. AnovaOne Way com teste complementar Post Hoc de Tukey avaliou diferença das variáveis nos três grupos

Conexões: revista da Faculdade de Educação Física da UNICAMP, Campinas, v. 10, n. 1, p. 38-49, jan./abr. 2012. 
analisados. Na intenção de se manter a cientificidade da pesquisa, o presente estudo admitiu o nível de significância de $\mathrm{p}<0,05$. Os resultados foram obtidos no proprama SPSS 16.0 para Windows.

\section{RESULTADOS}

O valor médio correspondente ao consumo máximo de oxigênio para G1 foi de 45,28 $\pm 2,01$ $\mathrm{ml} / \mathrm{kg} / \mathrm{min}$ para G2 foi de 49,07 $\pm 2,26 \mathrm{ml} / \mathrm{kg} / \mathrm{min}$ e para G3 de 45,62 $\pm 1,76 \mathrm{ml} / \mathrm{kg} / \mathrm{min}$. A descrição do $\mathrm{VO}_{2}$ máx nos grupos estudados pode ser vista na Figura 1. Os dados de $\mathrm{VO}_{2}$ máx assumiram distribuição normal ( $\mathrm{p}>0,05)$. Encontrou-se diferença significativa no $\mathrm{VO}_{2}$ máx de acordo com o posicionamento em campo $(\mathrm{p}=0,001)$. Diferença no $\mathrm{VO}_{2}$ máx entre $\mathrm{G} 1$ e $\mathrm{G} 2$ ( $\mathrm{p}$ $=0,002)$ e entre $\mathrm{G} 2$ e $\mathrm{G} 3(\mathrm{p}=0,003)$. Não foi encontrada diferença no $\mathrm{VO}_{2}$ máx entre $\mathrm{G} 1$ e $\mathrm{G} 3$ $(\mathrm{p}=0,938)$. Não foi encontrada diferença significativa entre os grupos para as variáveis: idade $(\mathrm{p}=0,18)$, altura $(\mathrm{p}=0,09)$, massa corporal $(\mathrm{p}=0,11), \mathrm{IMC}(\mathrm{p}=0,17)$ e $\% \mathrm{G}(\mathrm{p}=0,49)$.

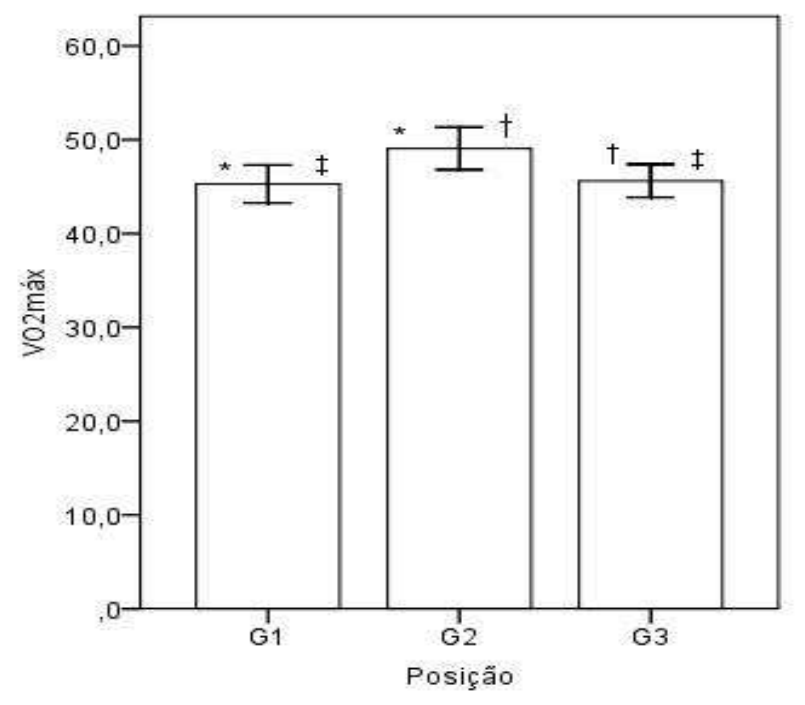

Figura 1. Consumo máximo de oxigênio dos jogadores de futebol de acordo com seu posicionamento em campo medido através do teste de corrida de vai-e-vem apresentados através da média \pm desvio padrão.

\section{DISCUSSÃO}

$\mathrm{O}$ presente estudo teve por objetivo comparar o $\mathrm{VO}_{2}$ máx de jogadores juvenis de futebol em

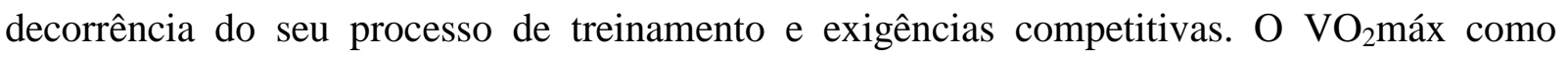
referência da condição aeróbica indica a possibilidade de manutenção de certa qualidade de

${ }^{*} p=0,002 ; \dagger p=0,003 ; \ddagger p=0,938$

Conexões: revista da Faculdade de Educação Física da UNICAMP, Campinas, v. 10, n. 1, p. 38-49, jan./abr. 2012. 
jogo expressa na densidade dos estímulos (relação esforço-recuperação). ${ }^{18}$ Pode-se afirmar que os atletas em $\mathrm{G} 2$ obtiveram $\mathrm{VO}_{2}$ máx significativamente superiores ao do G1 e G3.Esses resultados corroboram com o estudo de Rocha ${ }^{19}$ com jogadores juvenis e difere-se dos obtidos por Burger-Mendonça et al., ${ }^{20}$ que não encontraram distinção no $\mathrm{VO}_{2}$ máxentre jogadores coreanos juvenis. Os autores acreditam que a ausência de um treinamento especializado justifique essa semelhança nos valores de consumo de oxigênio. Balikian et al. ${ }^{21}$ não encontraram diferença no limiar anaeróbio e no consumo máximo de oxigênio em decorrência do posicionamento tático em campo. Vale salientar que o grupo estudado anteriormente foi de profissionais, diferente dos deste estudo, realizado com atletas juvenis. Destaca-se ainda a diferença no protocolo de teste. Alguns estudos utilizam esteira ergométrica em laboratório para avaliar o consumo de oxigênio, entretanto o presente estudo realizou uma sessão de exercícios contínuos e progressivos até a exaustão. ${ }^{17}$ A grande vantagem da investigação específica no campo é a obtenção de dados preciosos para a equipe técnica, no sentido de rentabilizar, tanto quanto possível, o treino desses atletas. ${ }^{18}$

Os resultados indicaram que os zagueiros e atacantes possuem um $\mathrm{VO}_{2}$ máx inferior quando comparados aos meio campistas e laterais. Menor $\mathrm{VO}_{2}$ máx encontrado para os zagueiros e atacantes pode ser explicado pelo fato de essas posições terem funções táticas relacionadas a um setor restrito do campo. O setor defensivo para os zagueiros, cuja função é de marcação e/ou cobertura dos laterais e meio campistas, e o ofensivo para os atacantes, cuja obrigação se restringe a finalização e marcação da saída de bola próximo à meta da equipe adversária. Por outro lado, os meio campistas e laterais têm função tanto no ataque (armação e finalização de jogadas) como na defesa (marcação aos adversários), ocasionando movimentação ampla e constante pelo campo de jogo. ${ }^{22,23}$ Comparando a distância de corrida em campo, encontrou-se que zagueiros $(9,1 \mathrm{a} 9,6 \mathrm{~km})$ e atacantes $(10,5 \mathrm{Km})$ cobrem menor percurso do que meio campistas $(10,2 \mathrm{a} 11 \mathrm{~km}){ }^{1,24,25}$

Outra diferença entre as posições táticasé com relação ao tempo que ficam parados durante as partidas. Os jogadores de meio campo ficam parados cerca de 14\% do tempo total de jogo, significativamente menos que os zagueiros $(21,7 \%)$ e os atacantes $(17,9 \%){ }^{25}$ Percebe-se então, com os dados supracitados, que não somente a distância total percorrida em campo influencia o $\mathrm{VO}_{2}$ máx, mas também, a intensidade do estimulo exigido durante os jogos, já que 
os meio campistas percorrem grande parte da distância total do jogo correndo em baixa intensidade, além dos momentos comuns de alta intensidade dos atacantes e defensores. ${ }^{4,26}$ Os zagueiros e atacantes necessitam de maior potência e condicionamento anaeróbio, diferente dos meio campistas e laterais que tem solicitação energética alta e contínua. ${ }^{27,28}$ em seu estudo recente com jogadores do futebol francês apresentam que além dos jogadores do meio-campo correrem maior distância total na partida, as distâncias para as intensidades médias (entre 21$24 \mathrm{~km} / \mathrm{h}$ ) também são maiores se comparados aos defensores e atacantes.

A variabilidade do $\quad \mathrm{VO}_{2}$ máx entre futebolistas é grande. Não somente pelas características individuais, mas em virtude dos diferentes modelos e metodologias utilizadas nos treinamentos. ${ }^{11}$ Os atletas participantes destes estudos realizavam o treinamento de exercícios contínuos e intermitentes com o mesmo volume e intensidade relativa, caracterizando assim, um treinamento generalista. Parece, então, a partir das características de treinamento apresentadas, que as solicitações das competições são favoráveis a uma adaptação fisiológica específica à posição de jogo e à função tática, fazendo com que os meios campistas e laterais apresentem maior $\mathrm{VO}_{2}$ máx para este grupo de indivíduos avaliados.A movimentação específica dos jogadores durante as partidas desenvolve em G3 um condicionamento físico que atenda as suas necessidades competitivas. Como o treinamento não se diferenciava entre os grupos e o futebol é característico por um calendário longo e com muitas competições, acredita-se que estes estímulos, aliado aqueles provenientes do treinamento, foram suficientes para provocar adaptações impostas pelas exigências em campo.

Dados provenientes de outras investigações com jogadores de outras categorias, diferente nível de rendimento, época variada do treinamento e quantitativo de indivíduos avaliados, destaca-se importante como forma de maior conhecimento desta inter-relação entre treinamento e manifestação esportiva. A ratificação e complementação dos achados neste estudo podem servir de referência no processo de treinamento de atletas com diferentes níveis de rendimento, mas agrega conhecimento ao processo de formação de atletas no futebol competitivo para as categorias de base, visto que estes, geralmente, realizam um treinamento que não leva plenamente em consideração suas necessidades específicas da partida. 


\section{CONCLUSÃO}

A partir dos resultados obtidos, neste estudo, com atletas de futebol da categoria juvenil, conclui-se que os meio campistas e laterais apresentam maior $\mathrm{VO}_{2}$ máx em relação a jogadores das outras posições, mesmo que a condução do treinamento não privilegie diferenças em decorrência de sua função tática em campo. Isso se explica pela adaptação fisiológica indireta que ocorre como resultado das solicitações durante os jogos coletivos e competições.

\section{REFERENCIAS}

${ }^{1}$ EKBLOM, B. Applied physiology of soccer. Sports Medicine, Auckland, n.3, p. 50-60, 1993.

${ }^{3}$ RIENZI, E. et al. Investigation of anthropometric and work-rate profiles of elite South American international soccer players. Journal of Sports Medicine and Physical Fitness, Torino, v. 40, n. 2, p. 162-169, 2000.

${ }^{4}$ BANGSBO, J. BANGSBO, J.; MOHR, M.; KRUSTRUP, P. Physical and metabolic demands of training and match-play in the elite football player. Journal of Sports Sciences, London, v. 24, n. 7, p. 665-674, 2006.

${ }^{5}$ MORTIMER, L. et al. Comparação entre a intensidade do esforço realizada por jovens futebolistas no primeiro e no segundo tempo do jogo de futebol. Revista Portuguesa de Ciências do Desporto, Porto, v. 6, n. 2, p. 154-159, 2006.

${ }^{6}$ DI SALVO, V.; BARON, R.; TSCHAN, H.; CALDERON MONTERO, F. J.; BACHL, N.; PIGOZZI, F. Performance characteristics according to playing position in elite soccer. International Journal of Sports Medicine, Stuttgart, v. 28, n. 3, p. 222-227, 2007.

${ }^{7}$ BANGSBO, J. The physiology of the soccer--with special reference to intense intermittent exercise. Acta Physiologica Scandinavica: Supplementum, n. 619, p. 1-155, 1994. 
${ }^{10}$ LEAL JUNIOR, E. C. P. et al. Estudo comparativo do consumo de oxigênio e limiar anaeróbio em um teste de esforço progressivo entre atletas profissionais de futebol e futsal. Revista Brasileira Medicina do Esporte, São Paulo, v. 12, n. 6, p. 323-326, 2006.

${ }^{11}$ CAMPEIZ, J. M.; OLIVEIRA, P. R.; MAIA, G. B. M. Análise de variáveis aeróbia e antropométricas de futebolistas profissionais: Juniores e juvenis. Conexões: Revista da Faculdade de Educação Física da UNICAMP, v. 2, n. 1, p. 1-9, 2004.

${ }^{12}$ DENADAI, S. B. Índices fisiológicos de avaliação aeróbia: conceitos e aplicações. Ribeirão Preto: BSD, 1999.

${ }^{13}$ BANGSBO, J. BARROS NETO, T. L.; TEBEXRENI, A. S.; TAMBEIRO, V. L. Aplicações práticas da ergoespirometria no atleta. Revista da Sociedade de Cardiologia do Estado de São Paulo, São Paulo, v. 11, n. 3, p. 695-705, 2001.

${ }^{14}$ GARGAnTA, J. Performance energético-funcional no futebol. 1997. Disponível em: $<$ http \ www.marciofariacorrea.com.br>. Acesso em: 22 ago. 2009.

${ }^{17}$ DUARTE, M. F. S.; DUARTE, C. R. Validade do teste aeróbico de corrida de vai-e-vem de 20 metros. Revista Brasileira de Ciência e Movimento, Brasília, v. 9, n. 3, p. 07-14, 2001.

${ }^{20}$ BURGER-MENDONÇA, M. et al. Variáveis ventilatórias em jogadores Coreanos juvenis de futebol: comparação entre posições em campo. Movimento \& Percepção, Espírito Santo do Pinhal, v. 7, p. 178-190, 2007.

${ }^{21}$ BALIKIAN, P. et al. Consumo máximo de oxigênio e limiar anaeróbio de jogadores de futebol: comparação entre as diferentes posições. Revista Brasileira de Medicina do Esporte, São Paulo, v. 8, n. 2, p. 32-36, 2002.

${ }^{22}$ REILLY, T. Energetics of high-intensityexercise (soccer) with particular reference to fatigue. Journal of Sports Sciences, London, v. 15, p. 257-263, 1997. 
${ }^{23}$ ANJOS, R. Futebol: regras, esquemas táticos, posições e funções do goleiro ao pontaesquerda. São Paulo: Rumo, 1990.

${ }^{24}$ BANGSBO, J.; NORREGAARD, I.; THORSOE, F. Active profile of competition soccer. Canadian Journal of Sports Medicine, Canada, v. 16, p. 110-116, 1992.

${ }^{25}$ REILLY, T.; BANGSBO, J.; FRANKS, A. Anthropometric and physiological predispositions for elite soccer. Journal of Sports Sciences, London, 2000, v.18, p. 669-683.

${ }^{28}$ DELLAL, A.; WONG, D.; MOALLA, W.; CHAMARI, K. Physical and technical activity of soccer players in the french first league with special reference to their playing position. International SportMed Journal, v. 11, n. 2, p. 278-290, 2010. 